\title{
Role of Tumor Subtypes in Response to Neoadjuvant Chemotherapy in Non-metastatic Breast Cancer
}

\author{
Negar Mashoori, $\mathrm{MD}^{1 *}$; Ramesh Omranipour, $\mathrm{MD}^{2}$; Abdolali Assarian, $\mathrm{MD}^{3}$ \\ ${ }^{1}$ Tehran University of Medical Sciences, Tehran, Iran \\ ${ }^{2}$ Breast Disease Research Center, Department of Surgical Oncology, Cancer Institute, Imam Khomeini Hospital, Tehran University \\ of Medical Sciences, Tehran, Iran \\ ${ }^{3}$ Breast Surgery Unit, Imam Khomeini Hospital, Tehran University of Medical Sciences, Tehran, Iran
}

\begin{abstract}
Background: Neoadjuvant chemotherapy (NAC) is an integral part of breast cancer treatment. Determination of the factors that can distinguish patients who will have best response to NAC is invaluable. In this study, we aimed to elucidate the factors influencing patient response to NAC.

Methods: We retrospectively collected data of female patients with non-metastatic breast cancer that had received NAC followed by surgery, admitted to Imam Khomeini hospital between 2015-2019. We investigated the association between various tumor and patients' characteristics with pathologic complete response (PCR).

Results: Overall data of 205 female patients were collected. PCR was observed in $27.6 \%$ of cases. PCR rate in luminal A, luminal B, HER2 enriched, and TNB tumors was reported in $11.1 \%, 30.2 \%, 35.7 \%$, and $36.4 \%$ of patients respectively $(P=0.27)$. In patients with luminal B tumors, PCR was more prevalent in patients with positive HER2 only $(P=0.006)$. In our study factors which was significantly associated with PCR were: tumor grade, progesterone receptor (PR) status, and HER2 status. In the multiple regression model, positive PR in the tumor lowered the odds of pathologic response 3.6 times $(P=0.004)$.

Conclusion: In our study, tumor grade, PR status, and HER2 status was associated with response to NAC. PCR was more prevalent in non-luminal tumors; however, PCR rate in luminal B patients-especially those with HER2 positive status- was slightly less than non-luminals.

Keywords: Breast cancer, Neoadjuvant chemotherapy, Pathologic complete response, Tumor subtype

Cite this article as: Mashoori N, Omranipour R, Assarian A. Role of tumor subtypes in response to neoadjuvant chemotherapy in non-metastatic breast cancer. Arch Iran Med. 2021;24(12):881-886. doi: 10.34172/aim.2021.132
\end{abstract}

Received: June 3, 2020, Accepted: May 5, 2021, ePublished: December 1, 2021

\section{Introduction}

Breast cancer is the most prevalent site-specific malignancy among women worldwide..$^{1-3}$ In Iran, breast cancer is the most prevalent female malignancy, with an incidence of 31 per 100000 , accounting for $27 \%$ of all new cancer cases in females; it is the second cause of cancer-related deaths in this country with a mortality rate of 8.7 per 100000 in $2018 .{ }^{4}$

Neoadjuvant therapies (including chemotherapy) have been introduced in to breast cancer management since the $1970 \mathrm{~s}^{5}$ they were initially intended to downstage inoperable disease to make it operable, and subsequently their use extended to operable breast cancers, allowing less extensive surgery of the breast and axilla. ${ }^{5}$ Several benefits are proposed for neoadjuvant chemotherapy (NAC): the chance to observe the response of intact tumor to several agents ${ }^{2,6,7}$; providing some valuable prognostic information like pathologic complete response (PCR) to $\mathrm{NAC}^{2,3,6-10}$; probable tumor shrinkage, converting inoperable disease to operable or allowing the surgeon and patient to pursue less invasive surgeries., ${ }^{5,10}$ However, there are also some suggested drawbacks for neoadjuvant compared to adjuvant chemotherapy, such as the probable increased risk of local recurrence after breast conserving surgery due to inaccurate localization. ${ }^{5}$

Determining factors that can distinguish patients who will have the best response to NAC is invaluable, as it will allow unresponsive patients to undergo surgery without undue delay. Patients respond differently to NAC depending on various patient and tumor factors. Some of the most important factors that have been proposed to affect tumor response positively include: HER2-enriched and triple negative (TN) tumor subtypes, younger age, smaller tumor size; higher grade, presence of lymphovascular invasion, and high Ki67 level. ${ }^{1,3,5,6,9,10}$ In this study, we retrospectively collected the data of female patients with non-metastatic breast cancer who underwent NAC followed by surgery. The purpose of this study was to investigate the effect of different patient and tumor factors, especially tumor receptor status and tumor subtypes, on PCR to NAC.

\section{Materials and Methods}

\section{Patients and Treatment Characteristics}

Medical records of patients with breast cancer admitted to the Cancer Institute and breast surgery unit of Imam Khomeini hospital between 2015-2019 were reviewed 
retrospectively. The data was available only to research members, and no identification data of patients were revealed during research to unrelated members. Female patients with non-metastatic breast cancer at presentation who had received standard NAC followed by surgery were eligible for this study. Demographic data, clinical and surgery data, and pathologic tumor characteristics were collected.

\section{Tumor Characteristics}

Pre-NAC histologic characteristics of tumor according to core needle biopsy including immunohistochemistry (IHC) staining results, and pre-NAC pathologic status of regional lymph nodes, if they were sampled, were obtained. Tumors' receptor status was determined by IHC staining; estrogen receptor (ER) and progesterone receptor $(\mathrm{PR})$ were considered positive if $\geq 1 \%$ of tumor cells were stained. For HER2, a result of $3+$ was considered positive and 0 or $1+$ were considered negative; $2+$ results were further classified as positive or negative using FISH or CISH test. Regarding these IHC results, each patient's tumor subtype was classified as Luminal A (positive ER and/or PR and negative HER2 and Ki67 less than 15\%), Luminal B (positive ER and/or PR and positive HER2 and/ or Ki67 $\geq 15 \%$ ), HER2-enriched (negative ER and PR, and positive HER2), and TN (negative ER and PR and negative HER2). Luminal B tumors were further classified as HER2 positive only tumors, tumors with elevated Ki67 level, and tumors with both positive HER2 and elevated Ki67 level. Clinical stage at diagnosis was determined according to the guidelines of the American Joint Committee on Cancer Staging $7^{\text {th }}$ edition.

\section{Response to Treatment}

Most studies have defined PCR as absence of invasive tumor in previously involved breast and axilla; ${ }^{7}$ some have defined PCR as absence of invasive tumor in breast or axilla. ${ }^{6,9}$. In this study, to overcome the relatively small sample size, PCR was defined as the absence of invasive tumor in the breast tissue or regional lymph nodes; so, patients who had PCR only in breast or axilla were considered responders, too.

\section{Statistical Analysis}

To investigate the association between patient's and tumor's characteristics with PCR to NAC, statistical analysis was done using the chi-square or the Fisher's exact tests where necessary. Because we did not have any low grade or low stage tumors in the PCR group, we could not calculate chi-square for trend ( $\mathrm{MH}$ test) to compare stage and grade in PCR. Instead, we used the chi-square test to compare binary tumor grade or stage and PCR. Age and tumor size were compared between binary independent variables using independent $t$ test or Mann-Whitney $\mathrm{U}$ test considering Normality. $P$ value $\leq 0.05$ was considered significant.

Logistic regression was performed using the stepwise backward (likelihood ratio) method to analyze the independent variables associated with PCR. The alpha level for stepwise selection was considered to be 0.05 . Factors with a $P$ value less than 0.2 in univariate analyses were entered into the multiple regression model.

\section{Results}

\section{Patients and Tumors Characteristic}

Overall data of 205 female patients were collected and reviewed. The patients' mean age was $45.2 \pm 10.6$ (min:24, max:77) years. Detailed tumor characteristics are shown in Table 1. IHC and tumors subtypes results and their relation to other tumor variables are shown in Table 2.

\section{Chemotherapy and Surgery Data}

In patients with available data, the most commonly used chemotherapy regimen was standard courses of Adriamycin-cyclophosphamide-paclitaxel (67.8\%), followed by docetaxel-cyclophosphamide (15.3\%), and Adriamycin-cyclophosphamide (10.2\%). The adriamycincyclophosphamide-paclitaxel (ATC) regimen was delivered in two patterns of dose dense or conventional, and the most commonly used pattern was dose dense ATC. In patients with HER2 positive tumors, Herceptin was administered as part of neoadjuvant treatment.

Sixty-three percent of patients underwent mastectomy and $37 \%$ underwent breast conserving surgery. Regarding axillary staging, $66.7 \%$ of patients underwent axillary lymph node dissection and $33.3 \%$ underwent sentinel lymph node biopsy.

\section{Pathologic Data}

On pathological examination of surgical specimens, the most prevalent histology was invasive ductal carcinoma (IDC) (73.7\%). In $19 \%$ of cases, there was no residue of tumor. Table 3 depicts the pathological details of surgical specimens.

Table 1. Tumors Characteristics of Patients

\begin{tabular}{lccc}
\hline \multicolumn{1}{c}{ Tumor Characteristics } & Frequency & Percentage \\
\hline Histologic Subtype & IDC & 177 & $92 \%$ \\
& ILC & 8 & $4.2 \%$ \\
& Others & 7 & $37 \%$ \\
\hline & T1 & 30 & $17.6 \%$ \\
Pre-NAC Tumor size & T2 & 100 & $58.8 \%$ \\
& T3 & 21 & $12.4 \%$ \\
\hline Pre-NAC Nodal status & T4 & 19 & $11.2 \%$ \\
& N0 & 41 & $23.5 \%$ \\
\hline Pre-NAC Clinical stage & N1 & 137 & $74.3 \%$ \\
& N2 & 8 & $2.2 \%$ \\
\hline $\begin{array}{l}\text { Sonographic tumor size } \\
\text { (mm)- Median (IQR) }\end{array}$ & 1 & 6 & $3.7 \%$ \\
\hline IDC, Invasive ductal carcinoma; & & 115 & $71.4 \%$ \\
Neoadjuvant chemotherapy; IQR, Interquartile range. & & \\
\hline & 3 & 40 & $24.8 \%$ \\
\hline
\end{tabular}


Table 2. IHC and Tumors Subtype of Patients

\begin{tabular}{|c|c|c|c|}
\hline Tumor Characteristics & & Frequency & Percentage \\
\hline \multirow{4}{*}{$\mathrm{IHC}$} & ER positive & 113 & $68.9 \%$ \\
\hline & PR positive & 110 & $58.5 \%$ \\
\hline & HER2 positive & 80 & $42.6 \%$ \\
\hline & Ki 67 level & \multicolumn{2}{|c|}{ Median $(I Q R)=30(20-45)$} \\
\hline \multirow{2}{*}{ Overall tumor subtypes } & Luminal & 128 & $66.7 \%$ \\
\hline & Non-luminal & 64 & $33.3 \%$ \\
\hline \multirow{5}{*}{ Tumor subtypes } & Luminal A & 11 & $5.9 \%$ \\
\hline & Luminal B & 102 & $54.3 \%$ \\
\hline & HER2-enriched & 32 & $17 \%$ \\
\hline & TNBC & 28 & $14.9 \%$ \\
\hline & Not specified & 15 & $8 \%$ \\
\hline \multirow{3}{*}{ Luminal B Tumors } & HER2 positive only & 9 & $8.8 \%$ \\
\hline & Elevated Ki67 & 55 & $53.9 \%$ \\
\hline & Both HER2 positive and elevatedki67 & 38 & $37.3 \%$ \\
\hline \multirow{2}{*}{ Non-Luminal Tumors } & HER2 enriched & 32 & $53.3 \%$ \\
\hline & TNBC & 28 & $46.7 \%$ \\
\hline
\end{tabular}

IHC, Immunohistochemistry; Her2, human epidermal growth factor receptor 2ER, Estrogen receptor; PR, Progesterone receptor; TNBC, Triple negative breast cancer; Luminal A, Tumors which are positive for ER and/or PR and negative for HER2 and their Ki67 level is less than 15\%; Luminal B, Tumors which are positive for ER and/or PR and positive for HER2 and/or their Ki67 level is equal to or more than $\geq 15 \%$.

Table 3. Pathologic Details of Surgical Specimens

\begin{tabular}{|c|c|c|c|c|c|}
\hline \multicolumn{3}{|c|}{ Tumor Characteristics } & \multirow{2}{*}{$\begin{array}{c}\text { Frequency } \\
151\end{array}$} & \multirow{2}{*}{$\begin{array}{c}\text { Percentage } \\
73.7 \%\end{array}$} & \multirow{2}{*}{$\frac{\text { Mean }}{-}$} \\
\hline \multirow{3}{*}{ Histologic subtype } & IDC & & & & \\
\hline & No tumor & & 39 & $19 \%$ & - \\
\hline & Others & & 15 & $8.3 \%$ & - \\
\hline Tumor size (mm) & & & - & - & $21 \pm$ \\
\hline \multirow{3}{*}{ Tumor grade } & 1 & & 25 & $18 \%$ & - \\
\hline & 2 & & 76 & $54.7 \%$ & - \\
\hline & 3 & & 38 & $27.3 \%$ & - \\
\hline Number of excised LNs & & & - & - & Median (IQR) = $8(4-12)$ \\
\hline Number of involved excised LNs & & & - & - & Median $(\mathrm{IQR})=1(0-4)$ \\
\hline \multirow{2}{*}{ Permanent LN status } & Free & & 89 & $46.1 \%$ & - \\
\hline & Involved & & 104 & $53.9 \%$ & - \\
\hline \multirow{8}{*}{$\begin{array}{l}\text { IHC results according to } \\
\text { permanent } \mathrm{LN} \text { status }\end{array}$} & \multirow{2}{*}{ Mean Ki67 } & Positive LN & - & - & Median $(I Q R)=30(20-40)$ \\
\hline & & Negative LN & - & - & Median $(\mathrm{IQR})=30(20-50)$ \\
\hline & \multirow{2}{*}{ Positive ER } & Positive LN & 71 & $72.4 \%$ & - \\
\hline & & Negative LN & 55 & $64.7 \%$ & - \\
\hline & \multirow{2}{*}{ Positive PR } & Positive LN & 66 & $68.8 \%$ & - \\
\hline & & Negative LN & 39 & $47.6 \%$ & - \\
\hline & \multirow{2}{*}{ Positive HER2 } & Positive LN & 34 & $35.1 \%$ & - \\
\hline & & Negative LN & 41 & $49.4 \%$ & - \\
\hline
\end{tabular}

IDC, Invasive ductal carcinoma; IHC, Immunohistochemistry; LN, lymph node; ER, estrogen receptor; Her2, human epidermal growth factor receptor 2; PR, progesterone receptor. 


\section{Response to Treatment}

PCR was reported in 48 patients (27.6\%). The mean age of patients with and without PCR was not significantly different. Mean pre-NAC sonographic tumor size was lower in patients with PCR, although the difference was not significant. Patients with ER negative, PR negative, and HER2 positive tumors had higher PCR in comparison to ER positive, PR positive, and HER2 negative tumors, respectively, although this difference was not significant in the ER group (ER: $P=0.09$, PR: $P=0.01$, HER2: $P=0.01$ ). Median Ki67 level was not significantly different between patients with and without PCR. PCR was more prevalent in non-luminal tumors than luminal ones $(P=0.23)$; In subgroup analysis, PCR in luminal A, luminal B, HER2 enriched, and TNB tumors was reported in $11.1 \%, 30.2 \%$, $35.7 \%$, and $36.4 \%$ of patients, respectively $(P=0.27$ respectively). In patients with luminal $B$ tumors, PCR was more prevalent in patients with positive HER2 only, followed by patients with both elevated Ki67 and positive
HER2 $(P=0.01)$. Table 4 shows detailed data of the relation between PCR and other variables.

In the multiple regression model, four factors with $P$ values ofless than 0.2 were entered in the backward logistic regression model, namely tumor size on sonography, ER, PR and HER2 status. We did not use the tumor grade in the multiple regression model, because we had only one grade type in the PCR group. The final backward model consisted of two variables: PR and tumor size. Positive PR lowered the odds of pathologic response by $72 \%$ (adjusted $\mathrm{OR}=0.28,95 \% \mathrm{CI}=0.12-0.67, P=0.004)$. The other factor (tumor size) did not affect PCR significantly (adjusted $\mathrm{OR}=0.98,95 \% \mathrm{CI}=0.95-1.01, P=0.10)$.

\section{Discussion}

The role of NAC as an integral part of breast cancer treatment and its inherent benefits have been largely confirmed. ${ }^{2,35-10}$ Determining a group of patients which will benefit most in terms of response and especially PCR,

Table 4. Detailed Data of Relation Between Pathologic Complete Response (PCR) and Other Variables

\begin{tabular}{|c|c|c|c|c|c|c|}
\hline & \multirow{2}{*}{ Variables } & \multicolumn{2}{|l|}{ PCR } & \multicolumn{2}{|c|}{ No PCR } & \multirow{2}{*}{$P$ value } \\
\hline & & Mean or Frequency & Percentage & Mean or Frequency & Percentage & \\
\hline \multicolumn{2}{|l|}{ Age (year) } & $44.02 \pm 9.8$ & - & $45.62 \pm 10.97$ & - & $0.43^{*}$ \\
\hline \multicolumn{2}{|c|}{ Sonographic tumor size (mm) } & Median (IQR) = $30(20-34)$ & - & $\begin{array}{c}\text { Median }(\mathrm{IQR})= \\
31(23-44.5)\end{array}$ & - & $0.11^{* *}$ \\
\hline \multirow{3}{*}{ Tumor stage } & 1 & 0 & $0 \%$ & 5 & $100 \%$ & \multirow{3}{*}{$0.40^{* * *}$} \\
\hline & 2 & 30 & $30.9 \%$ & 67 & $69.1 \%$ & \\
\hline & 3 & 8 & $25 \%$ & 24 & $75 \%$ & \\
\hline \multirow{3}{*}{ Tumor Grade } & 1 & 0 & $0 \%$ & 19 & $100 \%$ & \multirow{3}{*}{$0.01^{* * *}$} \\
\hline & 2 & 11 & $16.7 \%$ & 55 & $83.3 \%$ & \\
\hline & 3 & 0 & $0 \%$ & 31 & $100 \%$ & \\
\hline \multirow{2}{*}{ ER status } & Positive & 28 & $25 \%$ & 84 & $75 \%$ & \multirow{2}{*}{$0.09^{* * *}$} \\
\hline & Negative & 19 & $38 \%$ & 31 & $62 \%$ & \\
\hline \multirow{2}{*}{ PR status } & Positive & 18 & $20.2 \%$ & 71 & $79.8 \%$ & \multirow{2}{*}{$0.01^{* * *}$} \\
\hline & Negative & 27 & $39.8 \%$ & 41 & $60.3 \%$ & \\
\hline \multirow{2}{*}{ HER2 status } & Positive & 27 & $40.9 \%$ & 39 & $59.1 \%$ & \multirow{2}{*}{$0.01^{* * *}$} \\
\hline & Negative & 17 & $20 \%$ & 68 & $80 \%$ & \\
\hline Mean Ki67 & & $\begin{array}{c}\text { Median }(\text { IQR })=30 \\
\quad(20-47.5)\end{array}$ & - & $\begin{array}{c}\text { Median }(\text { IQR })=30 \\
(20-40)\end{array}$ & - & $0.83^{* *}$ \\
\hline \multirow{2}{*}{ Tumor Subtype } & Luminal & 28 & $26.2 \%$ & 79 & $73.8 \%$ & \multirow{2}{*}{$0.23^{* * *}$} \\
\hline & Non-luminal & 19 & $35.2 \%$ & 35 & $64.8 \%$ & \\
\hline \multirow{4}{*}{ All subtypes } & Luminal A & 1 & $11.1 \%$ & 8 & $88.9 \%$ & \multirow{4}{*}{$0.27^{* * *}$} \\
\hline & Luminal B & 26 & $30.2 \%$ & 60 & $69.8 \%$ & \\
\hline & HER2-enriched & 10 & $35.7 \%$ & 18 & $64.3 \%$ & \\
\hline & TNBC & 8 & $36.4 \%$ & 14 & $63.6 \%$ & \\
\hline \multirow{3}{*}{ Luminal B subtype } & Elevated Ki67 only & 9 & $18.4 \%$ & 40 & $81.6 \%$ & \multirow{3}{*}{$0.01^{* * *}$} \\
\hline & Positive HER2 only & 5 & $71.4 \%$ & 2 & $28.6 \%$ & \\
\hline & Elevated Ki67and positive HER2 & 12 & $40 \%$ & 18 & $60 \%$ & \\
\hline
\end{tabular}

IQR, Interquartile range; Luminal A, Tumors which are positive for ER and/or PR and negative for HER2 and their Ki67 level is less than 15\%; Luminal B, Tumors which are positive for ER and/or PR and positive for HER2 and/or their Ki67 level is equal to or more than $\geq 15 \%$; ER, estrogen receptor; Her2, human epidermal growth factor receptor 2; PR, progesterone receptor.

"Independent $t$ test. "*Mann-Whitey $U$ test. ${ }^{* * *}$ Chi square test. 
is a key point in this regard. PCR rates of 11 to $37.9 \%$ have been reported in various studies. ${ }^{7,10-13}$ In two studies in our country, PCR rat of $32.9 \%$ and $52.7 \%$ were reported, ${ }^{1,6}$ which are higher than PCR in our study (27.6\%). It should be noted that the difference in PCR definition in our work (as described in the Methods section) should be considered when comparing our results with similar studies.

Multiple investigations have proved PCR as a prognostic marker of better outcome in breast cancer patients, and PCR is suggested to serve as a surrogate marker for overall survival. ${ }^{6,7,9,12-14}$

Many researches have evaluated the role of various tumor and patient factors on PCR. Multiple studies have postulated that PCR rate is significantly influenced by various tumor subtypes; most of them have reported that PCR rate is more pronounced in TN and HER2 positive tumors, and less considerable in luminal ones, with luminal A tumors shown to be the least responsive. . $^{3,6,9-13,15}$ Different studies have reported PCR rates of 20.4-60\% in triple negative breast cancer (TNBC) patients and 15.6-65\% in HER2 enriched tumors. ${ }^{7-12,14,16,17}$ The PCR rate for luminal $\mathrm{B}$ tumors has been reported to be $4.2-$ $15.4 \%$ in HER2 negative cases and $16-32 \%$ in HER2 positive ones. ${ }^{7,9,10,15}$. Luminal A tumors were less likely to be treated with NAC with reported PCR rates of $0-9 \%$ in various reports. ${ }^{7,910,16,18}$ In a study by Livinston-Rosanoff et $\mathrm{al}^{19}$ on 38864 patients, TN and HER2 positive tumors had better PCR in comparison to $\mathrm{HR}+/ \mathrm{HER}$ - tumors. In a prospective multicenter clinical trial by Boughey et al, ${ }^{10}$ PCR rates of $38.2 \%, 45.4 \%$, and $11.4 \%$ were reported in TN, HER2 positive, and HR positive HER2 negative tumors, respectively. In a study by Kim et al, ${ }^{15}$ it was shown that luminal B tumors are relatively resistant to NAC, and luminal B HER2 negative tumors had a significantly reduced clinical response and PCR in comparison to HER2-enriched and TN tumors.

A study by Sasanpour et $\mathrm{al}^{6}$ on 207 Iranian patients showed that HR positive status was associated with poorer response to NAC; also, in another retrospective study by Firouzabadi et $\mathrm{al}^{20}$ in Iran, it was shown that HER2 positive/ER negative tumors have a better response to NAC. In another study by Omranipour et $\mathrm{al}^{21}$ in the Iran Cancer Institute on 314 patients with ER positive HER2 negative tumors, overall PCR was observed in $14.6 \%$ of patients.

The independent influence of Ki67 level on response to NAC has not been investigated much; in a report by Kim et $\mathrm{al},{ }^{15}$ it was shown that in HER2 negative luminal tumors, increased Ki67 level is associated with increased response. In a study by Omranipour et $\mathrm{al}^{21}$ a cut-off level of $22.5 \%$ for Ki67 expression was determined for PCR prediction in ER positive HER2 negative tumors. In a study by Asano et $\mathrm{al}^{14}$ on TNBC patients, increased Ki67 level was associated with greater PCR.

In our study, grade 2 , negative $\mathrm{PR}$, and positive HER2 status was significantly associated with greater PCR. Tumors with PCR to NAC were more ER and PR negative, although the difference was not significant for ER status. In the logistic regression analysis, positive PR status lowered the odds of pathologic response by $72 \%$ (adjusted $\mathrm{OR}=0.28,95 \% \mathrm{CI}=0.12-0.67, P=0.004)$. HER2 positive tumors had more PCR. The median Ki67 level was not significantly different among responders and nonresponders; so, it seems that Ki67 level did not affect the patients' response to NAC in our study. In concordance with other reports, our findings showed that PCR was more observed in non-luminal tumors than luminal ones: the greatest response was seen in TN tumors followed by HER2-enriched and luminal B tumors; luminal A tumors had the least PCR rate, although the reported difference was not statistically significant. PCR was not considerably different across two non-luminal subtypes. It is of note that the PCR rate of luminal B tumors was not substantially different from non-luminal ones in our study. In luminal B tumors, HER2 positive only had remarkably more PCR (3.9 folds) in comparison to patients with elevated Ki67 levels only. Considering the findings of this study and the results of other reports, it can be concluded that in the era of targeted anti-HER2 therapy, positive HER2 status can reliably predict good response to NAC; also, in luminal B tumors, being HER2 positive might be a better predictor of response to NAC compared to high Ki67 levels.

In conclusion, in our study, factors which were significantly associated with PCR included tumor grade, PR status, and HER2 status; positive PR status lowered the odds of pathologic response by $72 \%$. In our patients, the most responsive subtypes were non-luminals; nevertheless, the PCR rate in luminal B patients was close to them, which shows NAC is an appropriate choice with expected good PCR in luminal B patients, especially those with positive HER2 status. These finding can be helpful in determining patients (especially those with luminal B tumors) who may benefit most from NAC.

The retrospective and non-randomized nature of this study along with its small sample size are the important drawbacks of this study. Moreover, defining PCR as the absence of invasive tumor in breast or regional nodes is another limitation which can cause difficulties in comparing the results of this study with similar ones. Randomized trials with more accurate data collection and larger sample sizes will be helpful to elucidate many of current controversies regarding the best patient selection method for NAC in breast cancer patients.

\section{Authors' Contribution}

We declare that all authors have contributed substantially in this study regarding design, data collection, analysis, and manuscript writing.

\section{Conflict of Interest Disclosures}

None to declare.

\section{Ethical Statement}

The Ethical board approval was waived regarding retrospective design of the study and data retrieval from medical records of 
patients with previous obtained permission for use in medical research. The data was available only to research members, and no identification data of patients were revealed during research to unrelated members.

\section{References}

1. Moazed V, Jafari E, Kalantari Khandani B, Nemati A, Roozdar A, Ben Razavi SA. Prognostic significance of reduction in Ki67 index after neoadjuvant chemotherapy in patients with breast cancer in Kerman between 2009 and 2014. Iran J Pathol. 2018;13(1):71-7.

2. Bagegni NA, Tao Y, Ademuyiwa FO. Clinical outcomes with neoadjuvant versus adjuvant chemotherapy for triple negative breast cancer: a report from the National Cancer Database. PLoS One. 2019;14(9):e0222358. doi: 10.1371/journal. pone. 0222358.

3. Kim HY, Kim TH, Yoon HK, Lee A. The role of neutrophillymphocyte ratio and platelet-lymphocyte ratio in predicting neoadjuvant chemotherapy response in breast cancer. J Breast Cancer. 2019;22(3):425-38. doi: 10.4048/jbc.2019.22.e41.

4. The Global Cancer Observatory. GLOBOCAN 2020. Available from: http://gco.iarc.fr/today/data/factsheets/populations/364iran-islamic-republic-of-fact-sheets.pdf. Accessed March 25, 2020.

5. Asselain B, Barlow W, Bartlett J, Bergh J, Bergsten-Nordström E, Bliss J, et al. Long-term outcomes for neoadjuvant versus adjuvant chemotherapy in early breast cancer: metaanalysis of individual patient data from ten randomised trials. Lancet Oncol. 2018;19(1):27-39. doi: 10.1016/s14702045(17)30777-5.

6. Sasanpour P, Sandoughdaran S, Mosavi-Jarrahi A, Malekzadeh M. Predictors of pathological complete response to neoadjuvant chemotherapy in Iranian breast cancer patients. Asian Pac J Cancer Prev. 2018;19(9):2423-7. doi: 10.22034/ арjср.2018.19.9.2423.

7. Chou HH, Kuo WL, Yu CC, Tsai HP, Shen SC, Chu CH, et al. Impact of age on pathological complete response and locoregional recurrence in locally advanced breast cancer after neoadjuvant chemotherapy. Biomed J. 2019;42(1):66-74. doi: 10.1016/j.bj.2018.10.007.

8. Swain SM, Tang G, Lucas PC, Robidoux A, Goerlitz D, Harris $\mathrm{BT}$, et al. Pathologic complete response and outcomes by intrinsic subtypes in NSABP B-41, a randomized neoadjuvant trial of chemotherapy with trastuzumab, lapatinib, or the combination. Breast Cancer Res Treat. 2019;178(2):389-99. doi: 10.1007/s10549-019-05398-3.

9. Rivas M, Acevedo F, Dominguez F, Galindo H, Camus M, Oddo $\mathrm{D}$, et al. The neutrophil to lymphocyte ratio predicts the response to neoadjuvant chemotherapy in luminal B breast cancer. Asian Pac J Cancer Prev. 2019;20(7):2209-12. doi: 10.31557/арjср.2019.20.7.2209.

10. Boughey JC, McCall LM, Ballman KV, Mittendorf EA, Ahrendt GM, Wilke LG, et al. Tumor biology correlates with rates of breast-conserving surgery and pathologic complete response after neoadjuvant chemotherapy for breast cancer: findings from the ACOSOG Z1071 (Alliance) prospective multicenter clinical trial. Ann Surg. 2014;260(4):608-14. doi: 10.1097/ sla.0000000000000924.

11. Asano Y, Kashiwagi S, Onoda N, Noda S, Kawajiri H, Takashima $\mathrm{T}$, et al. Platelet-lymphocyte ratio as a useful predictor of the therapeutic effect of neoadjuvant chemotherapy in breast cancer. PLoS One. 2016;11(7):e0153459. doi: 10.1371/ journal.pone.0153459.

12. Li Y, Yang D, Chen P, Yin X, Sun J, Li H, et al. Efficacy and safety of neoadjuvant chemotherapy regimens for triple-negative breast cancer: a network meta-analysis. Aging (Albany NY). 2019;11(16):6286-311. doi: 10.18632/aging.102188.

13. Díaz-Casas SE, Castilla-Tarra JA, Pena-Torres E, OrozcoOspino M, Mendoza-Diaz S, Nuñez-Lemus $M$, et al. Pathological response to neoadjuvant chemotherapy and the molecular classification of locally advanced breast cancer in a Latin American cohort. Oncologist. 2019;24(12):e1360-e70. doi: 10.1634/theoncologist.2019-0300.

14. Asano $\mathrm{Y}$, Kashiwagi $\mathrm{S}$, Onoda $\mathrm{N}$, Noda $\mathrm{S}$, Kawajiri $\mathrm{H}$, Takashima T, et al. Predictive value of neutrophil/lymphocyte ratio for efficacy of preoperative chemotherapy in triplenegative breast cancer. Ann Surg Oncol. 2016;23(4):1104-10. doi: 10.1245/s10434-015-4934-0.

15. Kim HS, Yoo TK, Park WC, Chae BJ. Potential benefits of neoadjuvant chemotherapy in clinically node-positive luminal subtype(-) breast cancer. J Breast Cancer. 2019;22(3):412-24. doi: 10.4048/jbc.2019.22.e35.

16. Cuello-López J, Fidalgo-Zapata A, López-Agudelo L, Vásquez-Trespalacios E. Platelet-to-lymphocyte ratio as a predictive factor of complete pathologic response to neoadjuvant chemotherapy in breast cancer. PLoS One. 2018;13(11):e0207224. doi: 10.1371/journal.pone.0207224.

17. Imeokparia FO, Hughes TM, Dossett LA, Jeruss JS, Chang $\mathrm{AE}$, Sabel MS. Axillary pathologic complete response in inflammatory breast cancer patients: implications for SLNB? Ann Surg Oncol. 2019;26(10):3374-9. doi: 10.1245/s10434019-07597-0.

18. O'Halloran N, Lowery A, Curran C, McLaughlin R, Malone C, Sweeney K, et al. A review of the impact of neoadjuvant chemotherapy on breast surgery practice and outcomes. Clin Breast Cancer. 2019;19(5):377-82. doi: 10.1016/j. clbc.2019.04.011.

19. Livingston-Rosanoff D, Schumacher J, Vande Walle K, Stankowski-Drengler T, Greenberg CC, Neuman H, et al. Does tumor size predict response to neoadjuvant chemotherapy in the modern era of biologically driven treatment? a nationwide study of US breast cancer patients. Clin Breast Cancer. 2019;19(6):e741-e7. doi: 10.1016/j.clbc.2019.05.014.

20. Firouzabadi D, Rezvani A, Dehghanian A, Mahmoudi L. Association of Ki67 and tumor marker p53 in locally advanced breast cancer patients and evaluation of response to neoadjuvant chemotherapy: a survey in South Iran. Cancer Manag Res. 2019;11:6489-97. doi: 10.2147/cmar.s203831.

21. Omranipour R, Jalili R, Yazdankhahkenary A, Assarian A, Mirzania M, Eslami B. Evaluation of pathologic complete response (pCR) to neoadjuvant chemotherapy in Iranian breast cancer patients with estrogen receptor positive and HER2 negative and impact of predicting variables on $\mathrm{pCR}$. Eur J Breast Health. 2020;16(3):213-8. doi: 10.5152/ejbh.2020.5487. 